\title{
Hsp70 Induction and $h s p 70$ Gene polymorphisms as Indicators of acclimatization under hyperthermic conditions
}

\author{
T.L. Kresfelder, N. Claassen and M.J. Cronjé
}

\begin{abstract}
The possibility of using Hsp70 and $h s p 70$ gene polymorphisms as markers of acclimatization was investigated. Volunteers (22) were subjected to an acclimatization regimen and blood analysed for Hsp70 (Hsp72) and hsp70 polymorphisms before and after a heat tolerance test. Physiological parameters denoting acclimatization, or not, were correlated to levels of Hsp70 and combination of $h s p 70$ genes. Only individuals that acclimatized had decreased basal Hsp70 levels and increased ability to induce Hsp70 together with a specific hsp70 genotype combination. We propose that Hsp70 levels (basal vs. induced) with the genotype combination have the potential to be used as markers of acclimatization.
\end{abstract}

\section{Article Outline}

1. Introduction

2. Materials and methods

2.1. Subjects

2.1.1. Preliminary procedures

2.1.1.1. Medical screening

2.2. Experimental testing

2.2.1. Exercise protocol

2.3. Blood analysis

2.3.1. Isolation of serum

2.3.2. Hsp70 quantification in the serum

2.3.3. DNA isolation and Polymerase chain reaction (PCR)

2.4. Statistical analysis

3. Results

3.1. Physiological parameters of the 22 volunteers

3.2. Hsp70 analysis

3.2.1. Representation of data following Hsp70 analysis

3.2.2. Hsp70 levels in serum of all individuals

3.3. hsp70 DNA polymorphisms

3.4. Hsp70 levels in individuals with genotype combination A/A, A1 A1, P2 P2

4. Discussion

4.1. Physiological parameters of the 22 volunteers

4.2. Hsp70 levels in serum of all individuals 
4.3. hsp70 DNA polymorphisms

4.4. Hsp70 levels in individuals with genotype combination A/A, A1 A1, P2 P2

4.5. Limitations

4.6. Summary

Acknowledgements

References

\section{Introduction}

Many occupational activities carried out in hot, humid conditions are plagued by problems related to heat stress. Such endeavors in South Africa include the Comrades Ultramarathon and the underground mining industry (Stewart, 1982; Steinmann and Bornman, 1996). One way of preventing such ailments involves proper acclimatization to hot conditions (Armstrong, 1998). Acclimatization is a process which occurs as a result of repeated mild increases in core temperature, which allows an organism to carry out work in the heat due to better heat dissipation (Moseley, 1997). The improved thermoregulatory ability is the result of adaptive physiological changes that occur in an individual as a result of repeated exposure to a stressful thermal environment (Armstrong, 1998).

Heat shock proteins (HSP) are stress proteins which are formed in cells due to various types of stimuli, which include heat, exercise and oxidative stress (Powers et al., 2001). The main function of HSP are to act as molecular chaperones, assisting with folding of newly synthesized proteins and refolding of partially denatured proteins (Forreiter and Nover, 1998). Specifically, the $70 \mathrm{kDa}$ family of HSP play an important part in cellular protection and adaptation during and following exposure to stressful events, including heat stress. Two prominent members of the Hsp70 family are Hsp72, the inducible form of Hsp70 and Hsp73, a constitutively synthesized protein also known as Hsc70.

Li and Werb (1982) reported that an increase in Hsp72 levels results in thermotolerance when mammalian cells are exposed in vitro to a heating episode. The induction of Hsp72, rather than Hsp73, has also been shown to coincide with the onset of acclimatization (Maloyan et al., 1999). Acclimatization refers to the adaptive responses of cells exposed to elevated temperatures, i.e. mild heat shock treatment conditions which enable cells to survive the effects of a subsequent temperature insult which would normally be lethal to the cells (Moseley, 1997).

Regulation of HSP expression occurs at the transcriptional level by the binding of one or more transcription factors, known as heat shock factors (HSFs), to the heat shock element (HSE) which is located in the promoter region of the hsp gene. In the unstressed state, HSF1 is present as an inactive monomer in the cytoplasm. Hsp70 binds to HSF1 under non-stressed conditions, forming the folded, inactive monomeric state. Denaturation of cellular proteins during stress causes the depletion of free Hsp70 through interactions with these denatured proteins. HSF1 is therefore released, resulting in the unfolding of HSF1, allowing trimerization and translocation to the nucleus (Pirkkala et al., 2001; Morimoto, 1998). 
It has been proposed that thermo-intolerance might be the result of mutations or polymorphisms in the genes that encode for the HSP (Steinmann and Bornman, 1996). The different $h s p 70$ genes include hsp70-1, hsp70-2 (3'utr and pst I) and hsp70-hom (Milner and Campbell, 1990). These three genes are HLA-linked and are found in the class III region of the major histocompatibility complex (MHC). They are situated 92 kilobases $(\mathrm{kb})$ telomeric of the $C 2$ gene (Sargent et al., 1989; Milner and Campbell, 1990) and the hsp70 loci lie $12 \mathrm{~kb}$ apart. The hsp70-hom gene is situated $\sim 4 \mathrm{~kb}$ telomeric to the hsp70-1 gene. Both the hsp 70-1 and hsp70-2 genes encode the primary heat-induced Hsp70 protein as an identical protein product (Hunt and Morimoto, 1985; Milner and Campbell, 1990). Milner and Campbell (1990) also reported no increase in hsp70hom mRNA levels after heat shock treatment. The polymorphic sites within the hsp $70-1$ gene are located at positions $-110,+120,+190$ and +438 . A tri-allelic polymorphism is found in this region, consisting of alleles $\mathrm{A}, \mathrm{B}$ and $\mathrm{C}$. With gel electrophoresis, each allele is represented by a different fragment, identified according to the various mobilities as slow (A), fast (B) and intermediate $(\mathrm{C})$. The differences between these alleles originate at positions -110 and +120 (Milner and Campbell, 1992; Cascino et al., 1993a and Cascino et al., 1993b). The pst I restriction site, located on chromosome 6, lies within the hsp70-2 gene (Goate et al., 1987; Milner and Campbell, 1990). This is the polymorphic site used in analysing variations in the hsp70-2 gene by Pst I enzyme digests and restriction fragment length polymorphism (RFLP) analysis. Two fragments with lengths of $8.5 \mathrm{~kb}$ and $9.0 \mathrm{~kb}$ represent $h s p 70-2$ (L allele) and hsp70-2 (U allele) respectively (Goate et al., 1987; Favatier et al., 1997). In addition to this, a two-allele polymorphism exists within the $3^{\prime}$-untranslated region (3'utr) where each allele is represented by a $183 \mathrm{bp}$ and a $188 \mathrm{bp}$ fragment respectively. In heterozygous individuals, both of these fragments are visible together with a third heteroduplex fragment which migrates more slowly (Dressel and Günther, 1994). The presence of an Nco I site in the hsp70-hom gene coding sequence gives rise to two fragments of $1495 \mathrm{bp}$ and $550 \mathrm{bp}$ (Milner and Campbell, 1992).

The possibility of using Hsp70 and the $h s p 70$ gene polymorphisms as markers of acclimatization was investigated in this study. Volunteers were recruited and exposed to a heat stress episode (HTS test), then subjected to an acclimatization regimen followed by a second HTS test, whereafter serum from each individual was analysed for Hsp70 and hsp 70 polymorphisms. Physiological data obtained from each individual was correlated to the biochemical data in order to determine if a relationship exists between the levels of Hsp70 and the ability to acclimatize. Polymorphisms in the $h s p 70$ genes were also studied to determine whether a specific polymorphism contributed toward the ability to acclimatize.

\section{Materials and methods}

\subsection{Subjects}

Twenty-two individuals, all students at the University of Johannesburg (then Rand Afrikaans University, Johannesburg, South Africa) participated voluntarily in the study. Their anthropometric and gender traits were noted. The individuals refrained from any exercise or heat stress for three days before the exercise program. The ethics committee of the university approved this study, and all standard ethical procedures were complied with. 


\subsubsection{Preliminary procedures}

\subsubsection{Medical screening}

ECGs were conducted following the Bruce treadmill protocol, where each individual exercised up to $70 \%$ of their maximum heart rate to identify potential cardiovascular risks. Unfit volunteers were eliminated from the study.

\subsection{Experimental testing}

\subsubsection{Exercise protocol}

The exercise program was carried out over 7 days. On days 1 and 6, each individual completed the heat tolerance screening test (HTS) which consisted of a step test for $30 \mathrm{~min}$ at $28^{\circ} \mathrm{C}$ wet bulb temperature at an external work rate of $70 \mathrm{~W}$ (Kielblock and Schutte, 1998). The temperature-controlled facilities of Miningtek, a CSIR division, were used. The acclimatization program was performed over days 2 to 5 , consisting of a step test lasting $1 \mathrm{~h}$ at $28{ }^{\circ} \mathrm{C}$ wet bulb temperature. The external work rate each day consisted of combinations of $35 \mathrm{~W}$ or $70 \mathrm{~W}$ allocated in $15 \mathrm{~min}$ intervals. The increase from $35 \mathrm{~W}$ to $70 \mathrm{~W}$ was achieved by increasing the stepping rate to twice the speed used for $35 \mathrm{~W}$. Core (oral) temperatures were measured before and after every exercise session including before and after each HTS test, while heart rates were monitored throughout each exercise session. Sweat rate was determined by weighing each individual before and after exercise every day.

\subsection{Blood analysis}

\subsubsection{Isolation of serum}

Five milliliters of blood was collected in SST BD Vacutainer tubes and centrifuged at $1600 \times \mathrm{g}$ for 10 min at $17^{\circ} \mathrm{C}$. Serum was removed and stored at $-20^{\circ} \mathrm{C}$ until further use.

\subsubsection{Hsp70 quantification in the serum}

The StressXpress Hsp70 ELISA kit (Stressgen Biotechnologies, Victoria, BC, Canada) was used to determine the different levels (before HTS test=basal levels (C) and after HTS test induced levels (HS)) of the inducible form of Hsp70 (i.e. Hsp72, but referred to hereafter only as Hsp70) in the undiluted serum samples. The inducible Hsp70 protein standards were prepared by serial dilution from a stock solution of concentration $10 \mu \mathrm{g} / \mathrm{ml}$.

\subsubsection{DNA isolation and Polymerase chain reaction (PCR)}

DNA was extracted from whole blood samples of each volunteer with the Nucleon extraction and purification kit ${ }^{\mathrm{TM}}$ (BACC3, AEC Amersham, Buckinghamshire, UK). The hsp70-1, hsp 70-2 (3'utr and pst I) and hsp70-hom genes from $75 \mathrm{ng}$ DNA were amplified in an identical PCR cocktail but included specific primers for each gene $(0.125 \mu \mathrm{M})$. Taq polymerase $(0.625 \mathrm{U})$ was obtained from TaKaRa Biomedicals (Otsu, Shiga, Japan). 
The $h s p 70-1$ and $h s p 70-2$ (3'utr) PCR products were run on a $13.5 \%$ polyacrylamide gel (40\%; $37.5: 1)$ and a $9 \%$ polyacrylamide gel $(30 \% ; 29: 1)$ respectively, using a vertical Protean II XI Cell Electrophoresis apparatus (Biorad, Hercules, CA). The $h s p 70$ PCR products were treated with restriction digestive enzymes (RE), viz. hsp70-1 with Nci 1 (Promega, Madison, WI), hsp70-2 (pst I) with Pst I (Roche) and hsp70-hom with Ncol (Roche). The hsp70-1 digest product was run on a 1.8\% agarose gel while the hsp 70-2 (pst I) and hsp70-hom PCR products were run on a $0.8 \%$ agarose gel.

\subsection{Statistical analysis}

Statistical analysis was performed using SPSS 11 (SPSS Incorporated, 2000, Chicago, IL). We used ANOVA to compare the physiological parameters with all biochemical data. Standard error of mean (SEM) values were determined using Costat (Cohort, 1986, Berckley, CA) and $P<0.05$ was regarded as significant in all analysis, unless indicated otherwise. The linkage disequilibrium analysis was conducted using the Graphical Overview of Linkage Disequilibrium (GOLD), which was available at the website http://www.sciencedirect.com/science?_ob=RedirectURL\&_method=externObjLink\&_locator=u rl\&_cdi $=5104 \&$ plusSign=\%2B\&_targetURL $=$ http $\% 253 \mathrm{~A} \% 252 \mathrm{~F} \% 252 \mathrm{Fwww}$.well.ox.ac.uk $\% 25$ 2Fasthma\%252FGOLD. The LD was determined to examine whether a relationship existed between the presence or absence of certain $h s p 70$ genes.

\section{Results}

\subsection{Physiological parameters of the 22 volunteers}

The anthropometric and gender traits of qualified individuals are shown in Table 1. The two HTS tests, performed on days one and six, were carried out under identical conditions. The difference in core temperature before and after each HTS test on days 1 and 6 for each individual was the physiological parameter used to determine whether individuals were able to acclimatize (TT) or not (NT), as suggested by Kielblock and Schutte (1998). If the difference in core temperature was negative between days 1 and 6, individuals were grouped as TT. In contrast, individuals with unchanged core temperatures or a positive difference were grouped as NT. Fourteen individuals exhibited a decrease in core temperature (Fig. 1a: TT) while seven individuals experienced an increase in core temperature (Fig. 1b: NT). The data set for one individual was incomplete and is therefore not shown on the graph. 
Table 1.

Physical traits of volunteers who participated in the acclimatization program

\begin{tabular}{|c|c|c|c|c|}
\hline Volunteer & Age & Weight (kg) & Height (m) & Gender \\
\hline 1 & 28 & 60 & 1.65 & Female \\
\hline 2 & 20 & 62 & 1.60 & Female \\
\hline 3 & 28 & 65 & 1.72 & Male \\
\hline 4 & 20 & 59 & 1.62 & Female \\
\hline 5 & 20 & 105 & 1.98 & Male \\
\hline 6 & 26 & 72 & 1.61 & Female \\
\hline 7 & 20 & 57 & 1.64 & Female \\
\hline 8 & 28 & 66 & 1.65 & Female \\
\hline 9 & 19 & 60 & 1.63 & Female \\
\hline 10 & 26 & 66.5 & 1.71 & Female \\
\hline 11 & 28 & 47 & 1.52 & Female \\
\hline 12 & 35 & 73 & 1.87 & Male \\
\hline 13 & 21 & 71 & 1.85 & Male \\
\hline 14 & 19 & 65 & 1.78 & Female $^{\dagger}$ \\
\hline 15 & 26 & 61 & 1.74 & Female \\
\hline 16 & 26 & 43 & 1.54 & Female \\
\hline 17 & 23 & 47.5 & 1.60 & Female \\
\hline 18 & 25 & 89 & 1.74 & Female \\
\hline 19 & 25 & 68 & 1.62 & Female $^{\dagger}$ \\
\hline 20 & 22 & 61 & 1.65 & Female \\
\hline 21 & 20 & 47 & 1.55 & Female \\
\hline 22 & 24 & 75.5 & 1.79 & Male \\
\hline
\end{tabular}

Used contraceptives.

Luteal phase of menstrual cycle.

$\$$ Unable to determine whether able or unable to acclimatize as temperature was measured inaccurately. 


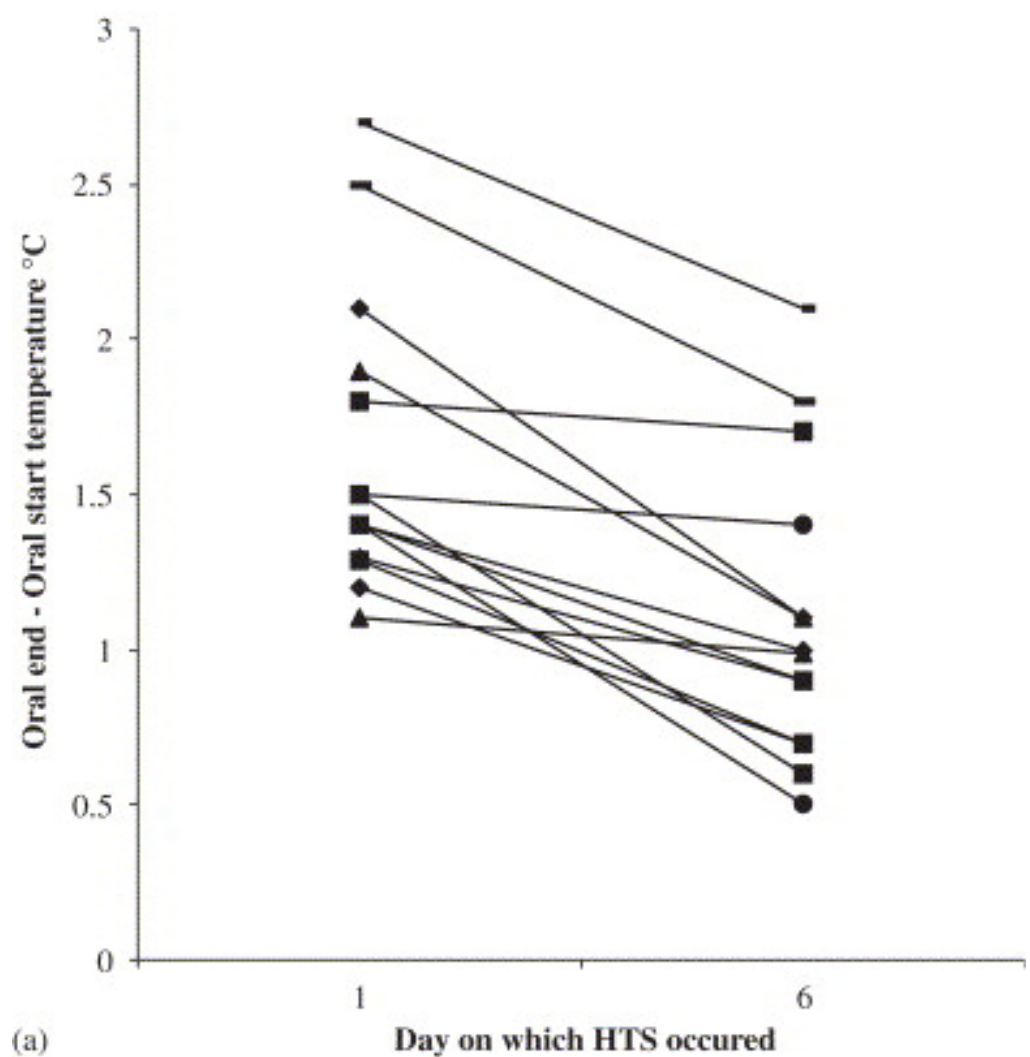

(a)

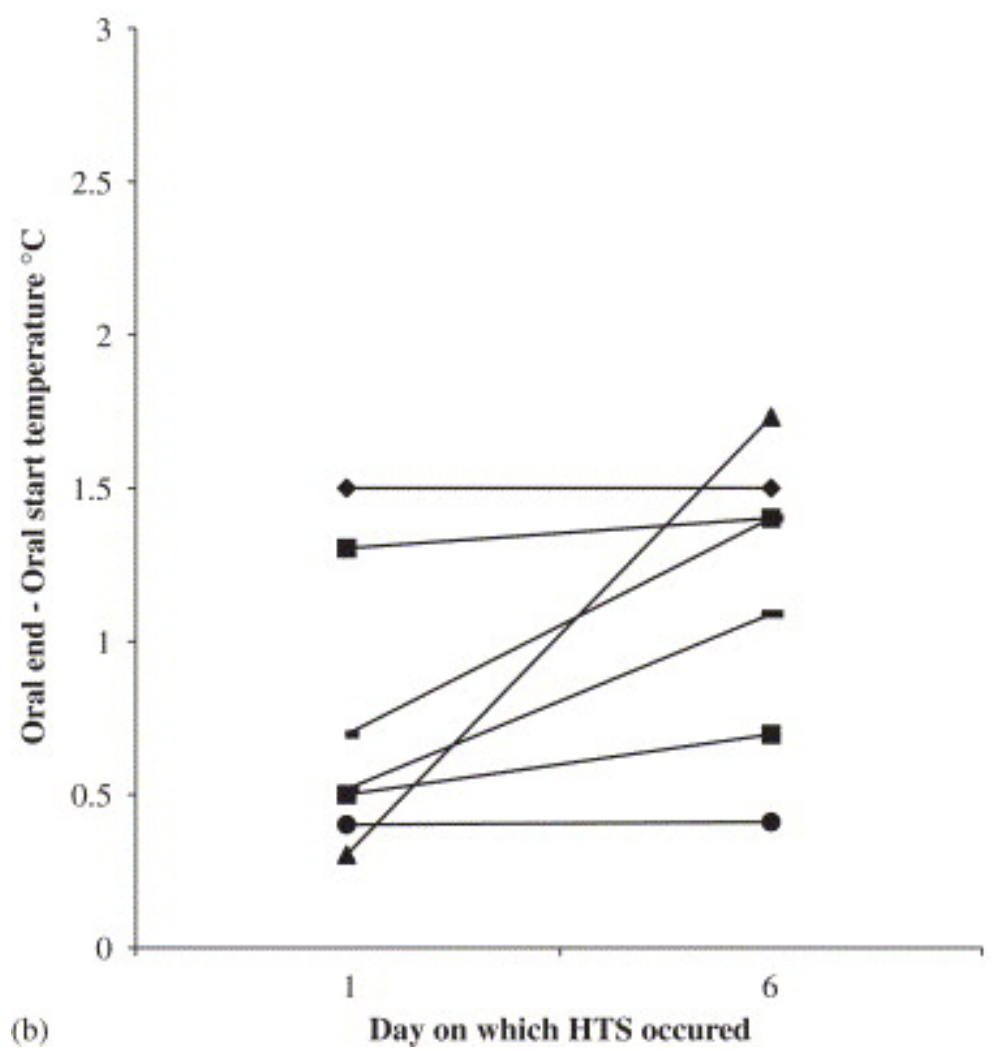


Fig. 1. Core temperature difference in individuals able to acclimatize (a; TT) or unable to acclimatize ( $b ; N T)$. The difference between the change in core temperature before and after each HTS test on days 1 and 6 was used to group the individuals. (a) These 14 individuals were considered to possess the ability to acclimatize as the difference was negative. (b) The individuals were grouped as NT as the difference in core temperature was either positive or zero.

\subsection{Hsp70 analysis}

\subsubsection{Representation of data following Hsp70 analysis}

The basal levels of the inducible form of Hsp70 (C1) were established in all the volunteers before they were subjected to the first HTS test on Day 1 (Pre-acclimatization). The increased levels of induced Hsp70 of all individuals were determined on Day 2 following the first HTS test (Preaccl, HS1). Individuals were then subjected to the acclimatization program (days 2 to 5), followed by the detection of Hsp70 levels before (C2) and after (HS2) the second HTS test on Day 6 (Post-acclimatization).

To compare the basal levels of inducible Hsp70 before the HTS (C1) with the elevated levels of inducible Hsp70 (HS1), we represented the data as percentage Hsp70 levels for all individuals in the TT group and NT group respectively (Pre-accl). We did the same after the acclimatization program (Post-accl, C2 vs. HS2). This allowed us to make allowance for inter-individual variation of Hsp70 levels between all the volunteers.

\subsubsection{Hsp70 levels in serum of all individuals}

In the TT individuals, the basal Hsp70 levels vs. induced Hsp70 levels were lower after the acclimatization program (Post-accl) compared to before the program (Pre-accl) (Fig. 2: TT). In contrast, the NT individuals had lower levels of induced Hsp70 after the acclimatization program compared to Pre-accl. (Fig. 2: NT). There was a significant decrease of HS1 relative to C1 in Preaccl TT individuals $(P<0.1)$, and in NT Post-accl individuals the difference between C2 and HS2 was significant at $P<0.05$. 


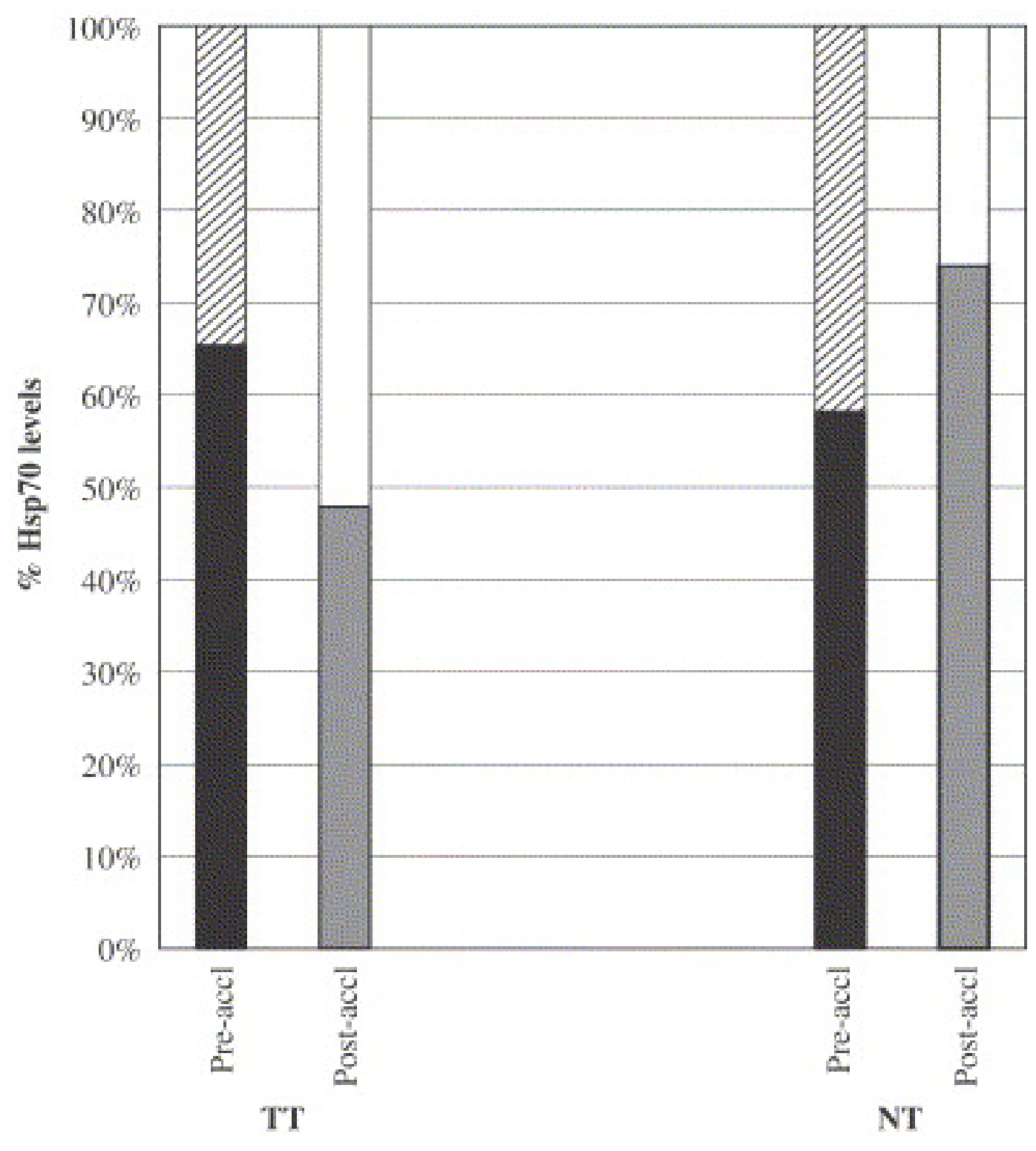

Fig. 2. Hsp70 levels (\%) in serum of individuals exhibiting the ability to acclimatize (TT) and the individuals who were unable to acclimatize (NT). Basal levels of Hsp70 in individuals before and after the acclimatization program (pre-accl and post-accl respectively) are represented by $\mathrm{C} 1$ and $\mathrm{C} 2 \square$. The induction of Hsp70 levels were determined after the HTS (heat tolerance screening) test both Pre-accl and Post-accl and are represented by HS1 Zand HS2 ᄃ. TT individuals showed decreased basal levels of Hsp70 Post-accl when compared to Pre-accl (C2 $\square_{x}$ vs. C1 $\mathbf{m})$ and a concomitant increase in HTS-induced levels of Hsp70 when compared to the Preaccl period (HS2 $\square$ vs. HS1 Z). In contrast, in NT individuals the increased levels of HTSinduced Hsp70 were significantly less following the acclimatization program (HS1 Zvv. HS2 $\square$ ) and the latter was associated with elevated basal levels of Hsp70 (C2 Ødiffered significantly from HS2 ᄃ, $P<0.05)$. 


\section{3. hsp70 DNA polymorphisms}

The $h s p 70$ DNA polymorphisms of all volunteers are shown in Fig. 3. Individuals 1, 2, 8, 10, 12 and 15 were all homozygous for allele $\mathrm{A}$, which exhibited the highest degree of curvature and was therefore retarded the most during migration through the gel, while individuals $3,6,7,9,13$ and 17 were all homozygous for allele $\mathrm{C}$ (Fig. 3a). The difference in migration between alleles $\mathrm{B}$ and $\mathrm{C}$ was minimal due to only a small difference in the degree of curvature. An Nci I restriction enzyme digest of $h s p 70-1$ PCR product was analysed for the presence of either allele B or allele $\mathrm{C}$ (Fig. 3b). All individuals showed an absence of the Nci I restriction site, indicating the presence of allele $\mathrm{C}$. Therefore individuals 4, 5, 11, 14, 16, 18, 19, 20, 21 and 22 were considered to be heterozygous for both alleles A and C. The hsp70-2 (3'utr) PCR product is shown in Fig 3c. Individuals $1,2,8,10,12$ and 15 were all homozygous for allele A1, which is $183 \mathrm{bp}$ in size while individuals 3, 6, 7, 9, 13 and 17 were all homozygous for allele A2 (188 bp). Individuals 4, $5,11,14,16,18,19,20,21$ and 22 were all heterozygous for both alleles A1 and A2. The third heteroduplex fragment was also visible, and migrated more slowly than the two alleles. The Pst I digest of hsp70-2 (pst I) PCR product is shown in Fig. 3d. Individuals 1, 2, 8, 10, 12, and 15 were homozygous for the absence of the pst I restriction site (P2 P2) resulting in a single fragment which was $1117 \mathrm{bp}$ in size. Individuals 3, 6, 7, 9, 13 and 17 were homozygous for the presence of the pst I restriction site (P1 P1). The larger of the two resulting fragments was $936 \mathrm{bp}$ in size, while the smaller fragment was $181 \mathrm{bp}$. Individuals 4, 5, 11, 14, 16, 18, 19, 20, 21 and 22 were heterozygous for the pst I restriction site (P1 P2), as the first allele possessed the pst I restriction site while this site was absent in the second allele. The hsp70-hom PCR product was digested with $N$ co I (Fig. 3e). Individuals 1, 3, 6, 7, 8, 9, 10, 11, 13, 16, 17, 19, 20, 21 and 22 were homozygous for the presence of the Nco I restriction site (N1 N1). The larger of the two fragments was $1495 \mathrm{bp}$ in size while the smaller fragment was $550 \mathrm{bp}$. Individuals 12 and 15 were homozygous for the absence of the Nco I restriction site (N2 N2) resulting in a single fragment (2045 bp). Individuals 2, 4, 5, 14 and 18 were heterozygous for the Nco I restriction site (N1 N2) as the first allele possessed this restriction site, while the second allele showed an absence of this restriction site. 

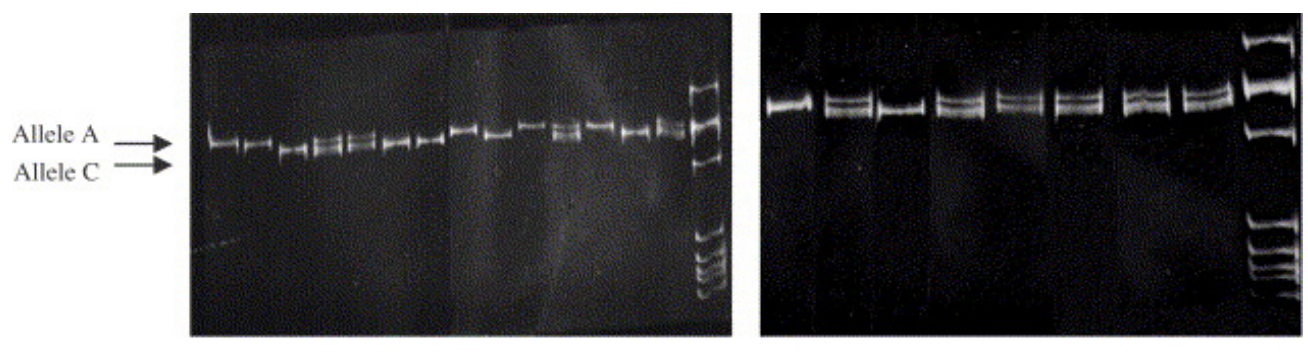

(a)

$\begin{array}{lllllllllllllll}1 & 2 & 3 & 4 & 5 & 6 & 7 & 8 & 9 & 10 & 11 & 12 & 13 & 14 & V^{*}\end{array}$
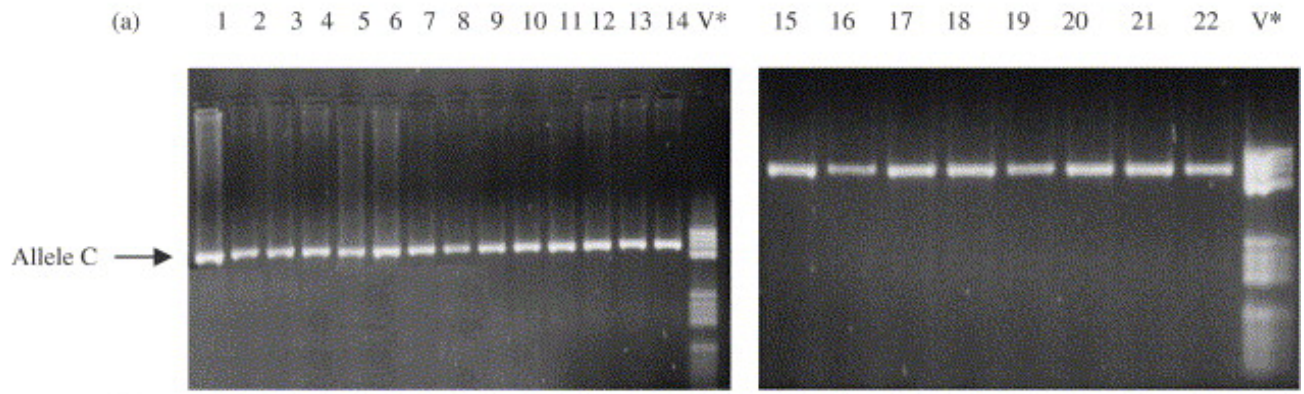

(b)
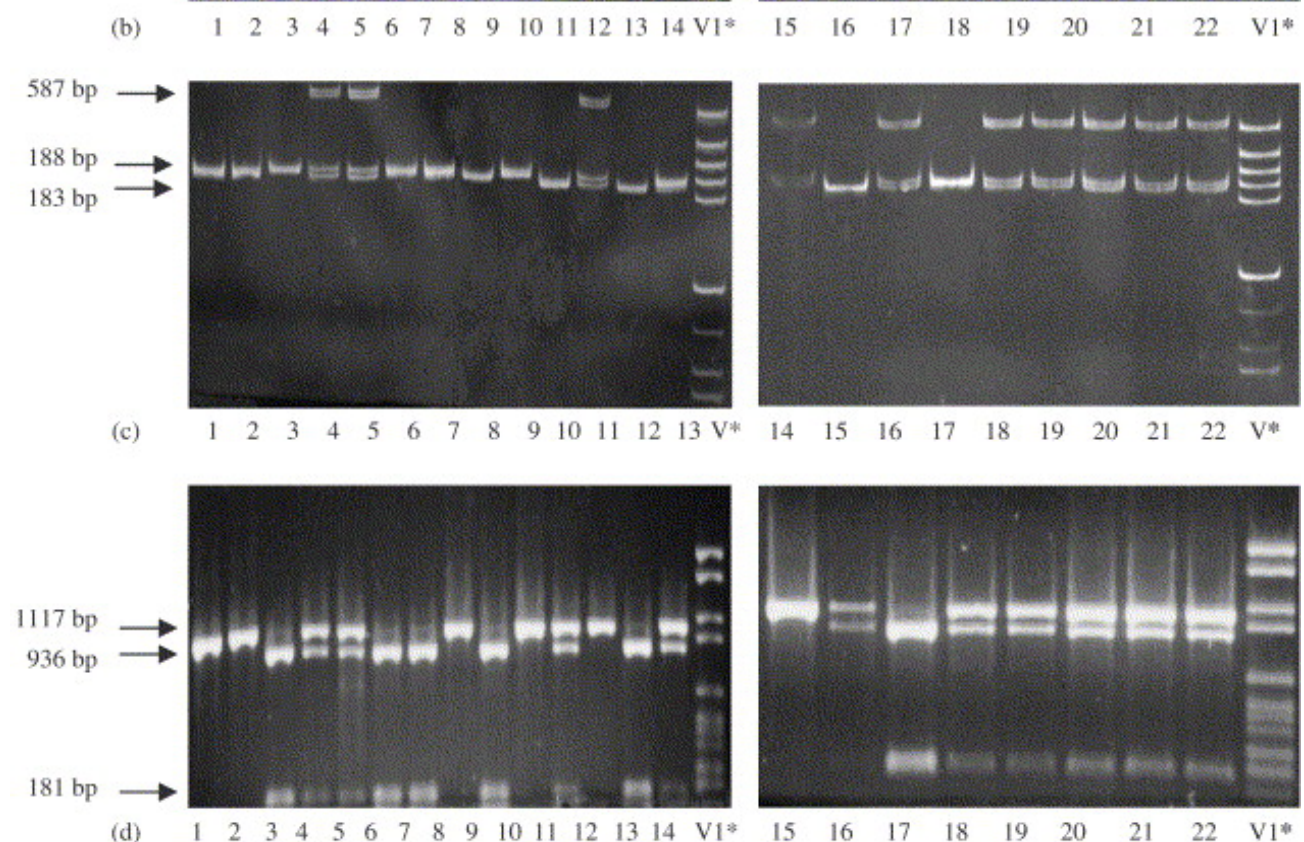

(d)
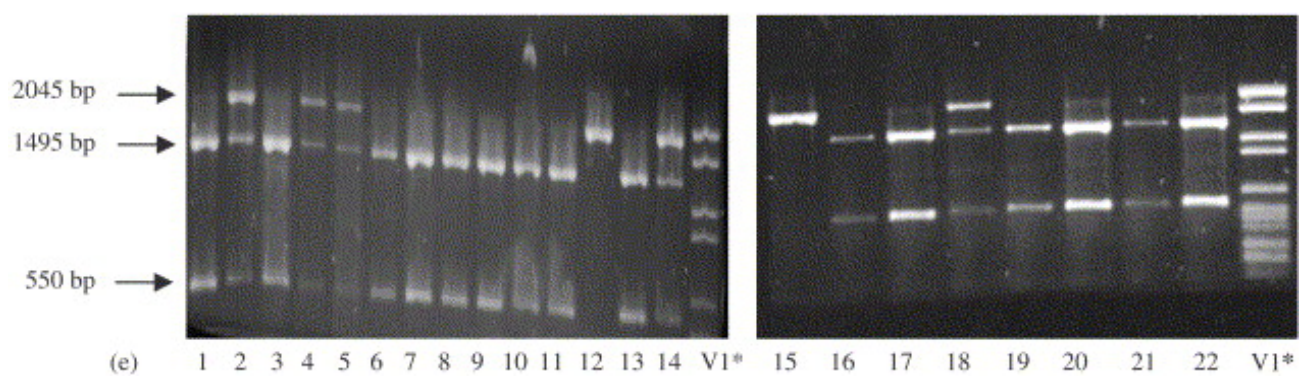

Fig. 3. DNA polymorphisms of all volunteers. (a) hsp70-1 polymorphisms: Individuals 1, 2, 8, 10,12 and 15 were all homologous for allele $\mathrm{A}$, while 3, 6, 7,9,13 and 17 were homozygous for allele C. (b) The presence of either allele $\mathrm{B}$ or $\mathrm{C}$ of the hsp70-1 polymorphism was determined by an Nci I RE digest of the PCR product. Individuals with no $\mathrm{Nci} I$ restriction site had allele $\mathrm{C}$ present. Individuals 4, 5, 11, 14, 16, 18, 19, 20, 21 and 22 were heterozygous for both alleles A and C. (c) hsp70-2 (3'utr) polymorphisms: Individuals 1, 2, 8, 10, 12 and 15 were all 
homozygous for allele A1 (183 bp), while 3, 6, 7, 9, 13 and 17 were homozygous for allele A2 (188 bp). Individuals 4, 5, 11, 14, 16, 18, 19, 20, 21 and 22 were heterozygous for both alleles A1 and A2. The heteroduplex fragment was also visible $(587 \mathrm{bp})$. MW marker $\mathrm{V}\left(\mathrm{V}^{*}\right), 8$ to $587 \mathrm{bp}$, was run with the $h s p 70-1$ and $h s p 70-2$ (3'utr) PCR products. (d) hsp70-2 (pst I) polymorphisms: Individuals $1,2,8,10,12$, and 15 were homozygous for the absence of the pst I restriction site (P2 P2) resulting in a single fragment (1117 bp), while 3, 6, 7, 9, 13 and 17 were homozygous for the presence of pstI (P1 P1; $936 \mathrm{bp}$ and $181 \mathrm{bp})$. Individuals 4, 5, 11, 14, 16, 18, 19, 20, 21 and 22 were heterozygous for the pst I restriction site (P1 P2; $1117 \mathrm{bp}, 936 \mathrm{bp}$ and $181 \mathrm{bp}$ ). (e) The Nco I digest of hsp70-hom PCR product: Individuals 1, 3, 6, 7, 8, 9, 10, 11, 13, 16, 17, 19, 20, 21 and 22 were homozygous for the presence of the Nco I restriction site (N1 N1; 1495 bp and 550 $\mathrm{bp}), 12$ and 15 were homozygous for the absence of the $N c o$ I restriction site (N2 N2; $2045 \mathrm{bp}$ ), while 2, 4, 5, 14 and 18 were heterozygous for the $N$ co I restriction site (N1 N2). MW marker VI (VI*) was run with the $\mathrm{Nci} I$, pst I and Nco I restriction digests on agarose gels, with fragments ranging from 154 to $2176 \mathrm{bp}$.

In order to confirm that the relationship between the alleles at linked loci is non-random (Jorde, 2000), we analysed the data for linkage disequilibrium (LD). We observed significant LD between the hsp70-1, hsp70-2 (3'utr) and hsp70-2 (pst I) genes (results not shown). Of the twenty-two individuals who participated in the exercise program, six were homozygous for the A/A allele (hsp70-1), the A1 A1 allele (3'utr) and the P2 P2 allele (pst I). Six of the individuals were homozygous for the $\mathrm{C} / \mathrm{C}$ allele (hsp70-1), the A2 A2 allele (3'utr) and the P1 P1 allele (pst I). The remaining ten individuals were heterozygous for the $\mathrm{A} / \mathrm{C}$ allele $(h s p 70-1)$, the A1 A2 allele (3'utr) and the P1 P2 allele (pst I).

The TT and NT groups were categorised according to the presence or absence of the different Hsp70 polymorphisms. This data is shown in Table 2. The six individuals who exhibited the A/A, A1 A1, P2 P2 genotype combination were all able to acclimatize, while none of the individuals who were unable to acclimatize showed this genotype combination. 
Table 2.

hsp70 DNA polymorphisms of individuals able (TT) or unable (NT) to acclimatize

\begin{tabular}{|l|l|l|l|}
\hline Hsp70 DNA polymorphisms & TT & $\boldsymbol{N T}$ & Total \\
\hline Hsp70-1 & & & \\
\hline A/A & 6 & 0 & 6 \\
\hline A/C & 5 & 5 & 10 \\
\hline C/C & 3 & 2 & 5 \\
\hline Hsp70-2 (3'utr) & & & \\
\hline A1 A1 & 6 & 0 & 6 \\
\hline A1 A2 & 5 & 5 & 10 \\
\hline A2 A2 & 3 & 2 & 5 \\
\hline Hsp70-2 (pst I) & & & \\
\hline P1 P1 & 3 & 2 & 5 \\
\hline P1 P2 & 5 & 5 & 10 \\
\hline P2 P2 & 6 & 0 & 6 \\
\hline Hsp70-hom & & & \\
\hline N1 N1 & 8 & 6 & 14 \\
\hline N1 N2 & 3 & 1 & 4 \\
\hline N2 N2 & 3 & 0 & 3 \\
\hline
\end{tabular}

\subsection{Hsp70 levels in individuals with genotype combination A/A, A1 A1, P2 P2}

We analysed the Hsp70 levels in individuals showing this specific genotype combination (abbreviated to AAP) (Fig. 4 +AAP) and in those where this genotype combination was absent (Fig. 4 -AAP). Basal levels of Hsp70 differed significantly $(P<0.05)$ to the HTS-induced Hsp70 levels both pre- and post-accl of the group showing the genotype combination. Furthermore, there was a significant reduction in basal Hsp70 levels in the +AAP group Post-accl when compared to those Pre-accl. In the group not having the genotype combination, basal levels increased significantly after acclimatization. In both cases (Pre- vs. Post- accl) HTS-induced Hsp70 differed significantly from the basal Hsp70 levels in the -AAP group. 


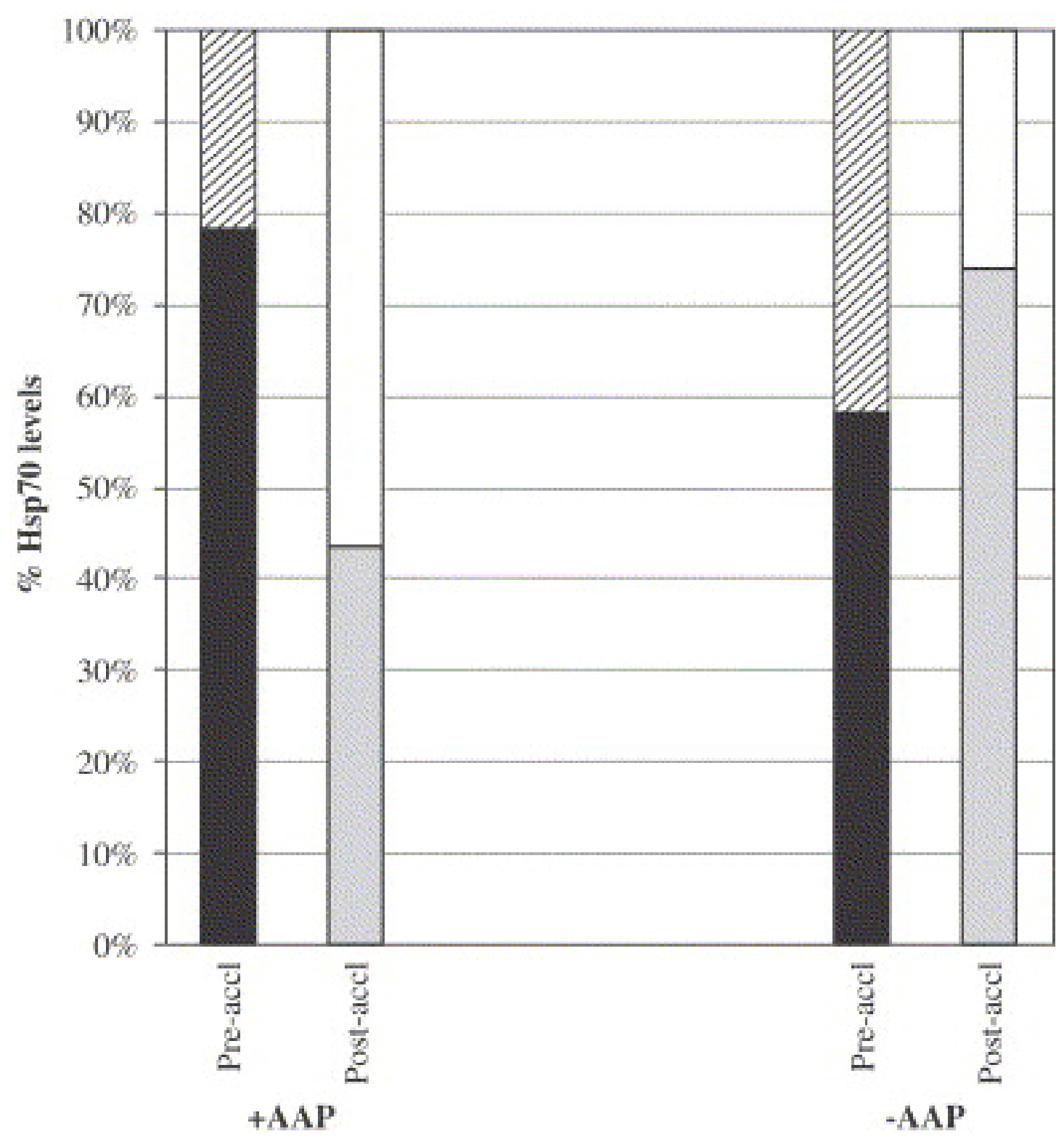

Fig. 4. Hsp70 levels (\%) in serum of individuals possessing the A/A, A1 A1, P2 P2 genotype combination (+AAP) and the individuals who did not possess this genotype combination (-AAP). Basal levels of Hsp70 differed significantly $(P<0.05)$ to the HTS-induced Hsp70 levels

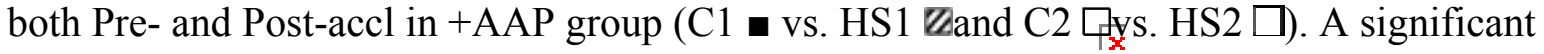
reduction in basal Hsp70 levels in the +AAP group Post-accl was observed when compared to those Pre-accl (C2 - vs. C1 $\mathbf{m})$. In the -AAP group basal levels increased significantly after acclimatization ( $\mathrm{C} 1$ - vs. C2 $\mathbf{m})$. In both cases (Pre- vs. Post- accl) HTS-induced Hsp70 differed significantly from the basal Hsp70 levels in the -AAP group (Pre-accl C1 — vs. HS1 Z; Post-accl C2 Фvs. HS2 ᄃ). 
When comparing the decrease in basal Hsp70 levels Post-accl, it is more significant in the +AAP group than in the TT group (Fig. 4: +AAP Post-accl). Therefore the reduction in basal Hsp70 levels following the acclimatization program between individuals possessing the AAP combination is 2-fold when compared to the TT group (Fig. 4: +AAP vs. Fig. 2 TT). Concomitantly, individuals possessing the genotype combination were able to induce much higher levels of HTS-induced Hsp70 after the acclimatization program (Fig. 4: +AAP Post accl) when comparing it to all TT individuals (Fig. 2: TT Post-accl).

\section{Discussion}

It is known that changes in physiological parameters, including alterations in core temperature and sweat rate as well as cardiovascular adjustments can be correlated to the ability to acclimatize to extreme temperatures (Armstrong, 1998). All organisms respond to stress factors such as heat exposure, strenuous exercise and environmental stressors (Powers et al., 2001) by inducing heat shock proteins (HSP), including Hsp70. It appears that variation in $h s p 70$ gene expression and polymorphisms are related to differential disease susceptibility and stress tolerance (Favatier et al., 1997). To our knowledge, the relationship between the ability to acclimatize and Hsp70 levels and $h s p 70$ polymorphisms are not known. Here we present data showing that only individuals who were able to acclimatize had decreased basal Hsp70 levels and elevated levels of induced Hsp70 after acclimatization together with a specific $h s p 70$ gene polymorphism.

\subsection{Physiological parameters of the 22 volunteers}

The fourteen individuals who showed a negative difference in core temperature between the two HTS tests on days 1 and 6 were considered to possess the ability to acclimatize (Fig. 1a). The seven individuals who demonstrated a positive difference or no difference in core temperature (Fig. 1b) between these two days were considered to be unable to acclimatize. Other studies have shown that thermoregulatory adaptations included increased sweat rate and earlier onset of sweat production resulting in decreased central body temperature, a response maximized after 5 days of heat acclimatization resulting from exercising in the heat (Armstrong, 1998; Pandolf, 1998). We were unable to correlate the change in sweat rate to their ability to acclimatize, most likely due to differences in fitness levels and non-uniformity in weight.

\subsection{Hsp70 levels in serum of all individuals}

In the TT group, the high basal levels of Hsp70 and low HTS-induced levels of Hsp70 (Fig. 2: TT Pre-accl C1 vs. HS1) agrees with findings of in vitro studies by Boshoff et al. (2000), who observed that increased basal Hsp70 levels in an individual resulted in lower amounts of Hsp70 able to be induced in response to heat stress in human monocytes. They concluded that the interspecies variation in stress protein expression, including Hsp70 in humans, may be caused by various factors including environmental (response to reactive oxygen species/ oxidative stress) or genetic (different HSF gene polymorphisms, leading to different pre-existing pools of HSF).

After exposing individuals to the acclimatization program, the basal Hsp70 levels were lower than before acclimatization (Fig. 2: TT C2 vs. C1). As a result of the decreased basal levels, the ability of the TT individuals to increase HTS-induced Hsp70 levels was increased after acclimatization (Fig. 2: TT C2 vs. HS2). 
In TT individuals, decreased induction of Hsp70 after heat shock may be due to pre-existing high basal levels of Hsp70 which can provide a pool of free Hsp70 to respond to the stress situation without further induction of Hsp70 being required. This may explain the decreased level of induced Hsp70 present in response to the first HTS test (Pre-accl, HS1). They responded to the second HTS test by inducing much higher levels of Hsp70 after the HTS test when compared to the lower C2 levels. It appeared as if the acclimatization program resulted in lowered basal levels of Hsp70, allowing these individuals to respond to the HTS test by inducing much higher levels of Hsp70. In the Zatsepina et al. (2000) study it was seen that the constitutive levels of Hsp70 in the mild-climate lizards were low, while HSF1 was present at high levels under non-stress conditions. This allowed for rapid and intense induction of HSP in response to heat stress, even after only brief exposure to a mild heat stress. Whether the acclimatization resulted in higher levels of HSF in our TT group remains to be determined.

Fehrenbach et al. (2000) observed that basal HSP expression was lower in trained (endurance runners) compared with untrained subjects. When applying an in vitro heat shock to resting blood samples, they observed higher increases in $h s p$ mRNA levels in the trained individuals. Shastry et al. (2002) investigated the Hsp70 levels in leukocytes after athletes exercised at 70\% VO2 max. They observed that pre-trained individuals had lower Hsp70 levels than untrained individuals. Also, no significant induction (but general increase) of Hsp70 was observed $15 \mathrm{~h}$ and $24 \mathrm{~h}$ after exercise in individuals who were trained. Therefore, trained individuals had lower basal Hsp70 levels and hardly expressed elevated Hsp70 levels when exercising. It is therefore likely that our acclimatization program, like the regular training, led to acclimatization, influencing the basal levels of pre-existing Hsp70.

The serum of NT individuals contained high basal (Pre-accl, C1) Hsp70 levels compared to the HS1 Hsp70 levels, which may be explained by the free pool of Hsp70 available to cope with the stress of the first HTS test. After the acclimatization program a larger amount of basal Hsp70 was present before the second HTS test (Post-accl, C2), which also may have prevented further Hsp70 induction in response to the second HTS test. Walsh et al. (2001) observed high levels of induced of Hsp70 (Hsp72) in serum after acute exercise that was accompanied with increased levels of plasma creatine kinase, a likely indicator of muscle tissue damage. Furthermore, it has been shown that constant exposure of eukaryotic cells to an extreme temperature $\left(43{ }^{\circ} \mathrm{C}\right)$ causes Hsp70 to be induced continuously (Morimoto, 1998). Whether the elevated serum levels of Hsp70 and inability of the NT group to acclimatize is related to tissue damage due to the acclimatization program, or the program perceived as "acute exercise" by these individuals, remains to be determined.

It is known that in leukocytes isolated from individuals exposed to an initial heat episode, which raised the core temperature to above $40{ }^{\circ} \mathrm{C}$, the level of Hsp70 induced in the isolated leukocytes in response to a second, in vitro heat shock decreased (Ryan et al., 1991). Therefore the induction of Hsp70 synthesis is inversely related to the level of protein in previously heat stressed thermotolerant cells. Similar to what was observed in these leukocytes, we observed that the relatively high basal levels of Hsp70 masked the effect of the HTS test on further induction of Hsp70.

Adaptation to heat is associated with enhanced physiological responses ultimately leading to better heat dissipation effectors and increased cardiovascular efficiency. There is evidence that heat-tolerant species, e.g. lizards (Zatsepina et al., 2000) and Turkmen (Lyashko et al., 1994) 
living in desert environments and rats heat-acclimated for 30 days (Maloyan et al., 1999) have adapted to high ambient temperatures by maintaining a higher level of Hsp70 and having an increased threshold temperature for induction of HSP, and in certain cases also showed faster kinetics of induction. However, the acquisition of thermotolerance through a short period in hot, humid environments combined with exercise cannot be compared to the evolutionary adaptation of living in hot desert climates. Although the physiological adaptation to heat shock is probably similar, long term adaptation of the heat shock response most likely is not. Our study consisted of a group of volunteers living in moderate environments, and being exposed for a short time to conditions specific to miners working underground or marathon athletes in South Africa. Therefore the acquisition of thermotolerance by these individuals was not similar to the long term adaptation of the desert-dwelling organisms. We therefore wanted to investigate whether the differential heat shock response between the TT and NT groups were associated with a specific Hsp70 polymorphism.

\section{3. hsp70 DNA polymorphisms}

The A/A, A1 A1, P2 P2 genotype combination occurred in approximately half of the TT group while none of the individuals in the NT group possessed this genotype combination. The most important aspect of the A/A, A1 A1, P2 P2 allelic combination seems to be the hsp 70-2 polymorphisms. The hsp70-1 and $h s p 70-2$ genes, which both encode identical Hsp70 protein products, express increased levels of mRNA after exposure to heat shock (Milner and Campbell, 1990). In a study carried out by Pociot et al. (1993), it was seen that the pst I polymorphism in the hsp 70-2 gene did not have an effect on the level of hsp70-2 mRNA. They did find that P1 P1 individuals expressed lower levels of mRNA than P2 P2 and P1 P2 individuals, but these differences were not significant. $h s p 70$ mRNA does not require heat stress to undergo translation, although in non-stressed cells the $h s p 70 \mathrm{mRNA}$ degrades more quickly than in stressed cells (Theodorakis and Morimoto, 1987). Under stress conditions the $h s p 70$ mRNA is more stable than under non-stress conditions.

\subsection{Hsp70 levels in individuals with genotype combination A/A, A1 A1, P2 P2}

When taking the Hsp70 levels of only the TT individuals exhibiting the A/A, A1 A1, P2 P2 genotype combination into account, the basal (C1) Hsp70 levels appeared to be higher than the Hsp70 levels in individuals where this genotype combination was absent (significant at $P<0.05$, Fig. 4: +AAP vs. -AAP), resulting in an increased ability to acclimatize. In fact, the C1 Hsp70 levels composed over $75 \%$ of the total amount of Hsp70 induced before the acclimatization program.

Therefore the presence of high basal (C1) Hsp70 levels together with the occurrence of the A/A, A1 A1, P2 P2 genotype combination may possibly be used as markers for the indication of acclimatization. The high Hsp70 induction after the second HTS test (HS2) may also possibly be used as an indication of the ability to acclimatize. It would, however, be far simpler, faster and more cost effective to obtain a blood sample in order to determine the $h s p 70$ genotype combination and the basal Hsp70 levels, as opposed to exposing the individual to two HTS tests, and an acclimatization program. 


\subsection{Limitations}

As this investigation was a pilot study, the sample group used was relatively small. Further studies should be carried out using larger sample groups, and care should be taken to use a more uniform group in terms of fitness levels and physiological parameters.

\subsection{Summary}

In this study, it was found that: (1) the basal Hsp70 levels in individuals who exhibited the ability to acclimatize decreased in response to the acclimatization program, while the ability of these individuals to respond to heat resulted in increased levels of induced Hsp70; (2) the individuals who were unable to acclimatize showed increased basal Hsp70 levels in response to acclimatization and decreased heat-induced Hsp70 levels; (3) the A/A, P2 P2 and A1 A1 genotype combination was only present in individuals who were able to acclimatize and absent in all individuals unable to acclimatize. These results suggest that the level of basal vs. induced Hsp70 in response to an acclimatization program and the presence or absence of the A/A, P2 P2 and A1 A1 genotype combination have the potential to be used as markers of acclimatization.

\section{References}

Armstrong, 1998 Armstrong, L. E., 1998. Heat acclimatization. Encyclopedia of Sports Medicine and Science.

http://www.sciencedirect.com/science?_ob=RedirectURL\&_method=externObjLink\&_locator=u rl\&_cdi $=5104 \&$ plusSign=\%2B\&_targetURL=http $\% 253 \mathrm{~A} \% 252 \mathrm{~F} \% 252 \mathrm{Fwww}$. sportsci.org $\% 252$ Fencyc\%252Findex.html $/$.

Boshoff et al., 2000 T. Boshoff, F. Lombard, R. Eiselen, J.J. Bornman, M. Bachelet, B.S. Polla and L. Bornman, Differential basal synthesis of Hsp70/Hsc 70 contributes to interindividual variation in Hsp70/Hsc70 inducibility, Cell. Mol. Life. Sci. 57 (2000), pp. 1317-1325.

Cascino et al., 1993a I. Cascino, R. Sorrentino and R. Tosi, Strong genetic association between HLA-DR3 and a polymorphic variation in the regulatory region of the $h s p 70-1$ gene, Immunogenetics 37 (1993), pp. 177-182.

Cascino et al., 1993b I. Cascino, S. D'Alfonso, N. Cappello, M. Giordano, A. Pugliese, Z. Awdeh, C.A. Alper and P.M. Richiardi, Gametic association of $h s p 70-1$ promoter region alleles and their inclusion in extended HLA haplotypes, Tissue Antigens 42 (1993), pp. 62-66.

Dressel and Günther, 1994 R. Dressel and E. Günther, A pentanucleotide tandem duplication polymorphism in the 3' untranslated region of the HLA-linked heat-shock protein 70-2 (HSP702) gene, Hum. Genet. 94 (1994), pp. 585-586.

Favatier et al., 1997 F. Favatier, L. Bornman, L.E. Hightower, E. Günther and B.S. Polla, Variation in $h s p$ gene expression and Hsp70 polymorphism: do they contribute to differential disease susceptibility and stress tolerance?, Cell Stress \& Chaperones 2 (1997), pp. 141-155. 
Fehrenbach et al., 2000 E. Fehrenbach, A.M. Niess, E. Schlotz, F. Passek, H.-H. Dickhuth and H. Northoff, Transcriptional and translational regulation of heat shock proteins in leukocytes of endurance runners, J. Appl. Physiol. 89 (2000), pp. 704-710.

Forreiter and Nover, 1998 C. Forreiter and L. Nover, Heat-induced stress proteins and the concept of molecular chaperones, J. Biosci. 23 (1998), pp. 287-302.

Goate et al., 1987 A.M. Goate, D.N. Cooper, C. Hall, T.K.C. Leung, E. Solomon and L. Lim, Localisation of a human heat-shock HSP70 gene sequence to chromosome 6 and detection of two other loci by somatic-cell hybrid and restriction fragment length polymorphism analysis, Hum. Genet. 75 (1987), pp. 123-128.

Hunt and Morimoto, 1985 C. Hunt and R.I. Morimoto, Conserved features of eukaryotic hsp70 genes revealed by comparison with the nucleotide sequence of human hsp70, Proc. Natl. Acad. Sci. USA 82 (1985), pp. 6455-6459.

Jorde, 2000 L.B. Jorde, Linkage disequilibrium and the search for complex disease genes, Genome Res. 10 (2000), pp. 1435-1444.

Kielblock and Schutte, 1998 Kielblock, A. J., Schutte, P. C., 1998. A guide to heat stress management. Johannesburg: Safety in Mines Research Advisory Committee Project Report GAP 505. Department of Minerals and Energy, Pretoria.

Li and Werb, 1982 G.C. Li and Z. Werb, Correlation between synthesis of heat shock proteins and development of thermotolerance in Chinese hamster fibroblasts, Proc. Natl. Acad. Sci. USA 79 (1982), pp. 3218-3222.

Lyashko et al., 1994 V.N. Lyashko, V.K. Vikulova, V.G. Chernicov, V.I. Ivanov, K.A. Ulmasov, O.G. Zatsepina and M.B. Evgen'ev, Comparison of the heat shock response in ethnically and ecologically different human populations, Proc. Natl. Acad. Sci. USA 91 (1994), pp. 1249212495.

Maloyan et al., 1999 A. Maloyan, A. Palmon and M. Horowitz, Heat acclimation increases the basal Hsp72 level and alters its production dynamics during heat stress, Am. J. Physiol. 276 (1999), pp. R1506-R1515.

Milner and Campbell, 1990 C.M. Milner and R.D. Campbell, Structure and expression of the three MHC-linked HSP70 genes, Immunogenetics 32 (1990), pp. 242-251.

Milner and Campbell, 1992 C.M. Milner and R.D. Campbell, Polymorphic analysis of the three MHC-linked HSP70 genes, Immunogenetics 36 (1992), pp. 357-362.

Morimoto, 1998 R.I. Morimoto, Regulation of the heat shock transcriptional response: cross talk between family of heat shock factors, molecular chaperones, and negative regulators, Genes Dev. 12 (1998), pp. 3788-3796. 
Moseley, 1997 P.L. Moseley, Heat shock proteins and heat adaptation of the whole organism, $J$. Appl. Physiol. 83 (1997), pp. 1413-1417.

Pandolf, 1998 K.B. Pandolf, Time course of heat acclimation and its decay, Int. J. Sports Med. 19 (1998), pp. S157-S160.

Pirkkala et al., 2001 L. Pirkkala, P. Nykänen and L. Sistonen, Roles of the heat shock transcription factors in regulation of the heat shock response and beyond, FASEB J. 15 (2001), pp. 1118-1131.

Pociot et al., 1993 F. Pociot, K.S. Ronningen and J. Nerup, Polymorphic analysis of the human MHC-linked heat shock protein 70 (Hsp70-2) and hsp70-hom genes in insulin-dependant diabetes mellitus (IDDM), Scan. J. Immunol. 38 (1993), pp. 491-495.

Powers et al., 2001 S.K. Powers, M. Locke and H.A. Demirel, Exercise, heat shock proteins, and myocardial protection form I-R injury, Med. Sci. Sports Exerc. 33 (2001), pp. 386-392.

Ryan et al., 1991 A.J. Ryan, C.V. Gisolfi and P.L. Moseley, Synthesis of 70 K stress protein by human leukocytes: effect of exercise in the heat, J. Appl. Physiol. 70 (1991), pp. 466-471.

Sargent et al., 1989 C.A. Sargent, I. Dunham, J. Trowsdale and R.D. Campbell, Human major histocompatibility complex contains genes for the major heat shock protein HSP70, Proc. Natl. Acad. Sci. USA 86 (1989), pp. 1968-1972.

Shastry et al., 2002 S. Shastry, D.O. Toft and M.J. Joyner, Hsp70 and Hsp90 expression in leukocytes after exercise in moderately trained humans, Acta Physiol. Scand. 175 (2002), pp. $139-146$.

Steinmann and Bornman, 1996 C.M.L. Steinmann and L. Bornman, Acclimatization, preconditioning and heat shock proteins, Med. Hypotheses 47 (1996), pp. 257-260.

Stewart, 1982 J.M. Stewart, Practical aspects of human heat stress. In: J. Burrows, Editor, Environmental Engineering in South African Mines, Cape and Transvaal Printers, Cape Town (1982).

Theodorakis and Morimoto, 1987 N.G. Theodorakis and R.I. Morimoto, Posttrancriptional regulation of Hsp70 expression in human cells: effects of heat shock, inhibition of protein synthesis and adenovirus infection on translation and mRNA stability, Mol. Cell. Biol. 7 (1987), pp. $4357-4368$.

Walsh et al., 2001 R.C. Walsh, I. Koukoulas, A. Garnham, P.L. Moseley, M. Hargreaves and M.A. Febbraio, Exercise increases serum Hsp72 in humans, Cell Stress Chaperones 6 (2001), pp. 386-393.

Zatsepina et al., 2000 O.G. Zatsepina, Kh.A. Ulmasov, S.F. Beresten, V.B. Molodtsov, S.A. Rybtsov and M.B. Evgen'ev, Thermotolerant desert lizards characteristically differ in terms of heat-shock system regulation, J. Exp. Biol. 203 (2000), pp. 1017-1025. 